\title{
Article
}

\section{Preoperative Planning for Superior Mesenteric Artery Aneurysm}

\author{
Cristiana Iulia Dumitrescu ${ }^{1}$, Catalin Ciobirca ${ }^{2}$, Radu Teodoru Popa ${ }^{2}$, Daniela Dumitrescu ${ }^{3, *}$, \\ Cornel Gheorghe Tambura ${ }^{4}$, Diana Maria Ciobirca ${ }^{5}$, Radu Stavaru ${ }^{6}$, Mihai Florin Tiuca ${ }^{7}$, Suzana Maces ${ }^{4,8}$, \\ Lucian Florentin Barbulescu ${ }^{2}$, Liliana Didi Popa ${ }^{2}$ and Sergiu Marian Cazacu ${ }^{9}$
}

1 Department of Pharmacology, University of Medicine and Pharmacy of Craiova, 200349 Craiova, Romania; dumitrescu.cristiana@gmail.com

2 Centrul Medical Speranta, Department of Cardiology, 200530 Craiova, Romania; catalin.ciobirca@yahoo.com (C.C.); popateodoru@yahoo.com (R.T.P.); lucian.barbulescu@dcti.ucv.ro (L.F.B.); lilianaciurezu@yahoo.com (L.D.P.)

3 Department of Radiology and Medical Imaging, University of Medicine and Pharmacy of Craiova, 200349 Craiova, Romania

4 SPAD IMAGING INTERNATIONAL Center Craiova, 200352 Craiova, Romania; tambura.cornel@gmail.com (C.G.T.); pielesuzana@yahoo.com (S.M.)

5 Slatina Emergency County Hospital, 230008 Slatina, Romania; diana.ciobirca@yahoo.com

6 Department of Cardiology, Craiova Emergency County Hospital, 200642 Craiova, Romania; radu_stavaru@yahoo.com

7 Vascular Department, Glan Clwyd Hospital, North Wales, Rhuddlan Rd, Bodelwyddan, Rhyl LL18 5UJ, UK; ftiuca@gmail.com

8 Doctoral School, University of Medicine and Pharmacy of Craiova, 200349 Craiova, Romania

9 Department of Internal Medicine 5th Year, University of Medicine and Pharmacy of Craiova,

check for updates

Citation: Dumitrescu, C.I.; Ciobirca, C.; Popa, R.T.; Dumitrescu, D.; Tambura, C.G.; Ciobirca, D.M.; Stavaru, R.;

Tiuca, M.F.; Maces, S.; Barbulescu, L.F.; et al. Preoperative Planning for Superior Mesenteric Artery Aneurysm. Appl. Sci. 2021, 11, 10311. https://doi.org/10.3390/app112110311

Academic Editor: Qi-Huang Zheng

Received: 29 September 2021

Accepted: 30 October 2021

Published: 3 November 2021

Publisher's Note: MDPI stays neutral with regard to jurisdictional claims in published maps and institutional affiliations.

Copyright: (c) 2021 by the authors. Licensee MDPI, Basel, Switzerland. This article is an open access article distributed under the terms and conditions of the Creative Commons Attribution (CC BY) license (https:/ / creativecommons.org/licenses/by/ $4.0 /)$.
200349 Craiova, Romania; cazacu2sergiu@yahoo.com

* Correspondence: daniela.dumitrescu@gmail.com

\begin{abstract}
Surgical procedures on various artery aneurysms are difficult to perform and require careful preparation. We have developed and now present in this paper a software platform, CardioCTNav, that can help in planning such procedures. The planning consists of a 3D rendering of the area of interest, virtual angiography, automated measurements, and virtual stent simulation.
\end{abstract}

Keywords: artery aneurysm; planning software; automated measurements; stent simulation

\section{Introduction}

A mesenteric artery aneurysm is an aneurysm that occurs in either the inferior or superior mesenteric arteries that irrigate visceral organs like the stomach. Mesenteric artery aneurysms are rare, with a prevalence of $5-8 \%$ of all aneurysms, and have an annual incidence of between $0.01 \%$ and $0.2 \%$ [1]. Superior mesenteric artery aneurysms are the third most common true visceral aneurysms, often affecting the proximal $5 \mathrm{~cm}$ of the artery [2] and accounting for $14 \%$ of all visceral aneurysms [3]. They occur predominantly in men who are in the 5th decade of life. Aneurysmal degeneration of the superior mesenteric artery does not occur frequently, but when it does, it may result in mesenteric ischemia or rupture [4], which can initiate a potentially life-threatening condition [3]. In about $25 \%$ of cases, visceral aneurysms present as surgical emergencies [1], although the disease is often asymptomatic. They are an incidental finding in almost half of the patients [5]. Around 22\% of patients with visceral aneurysms are diagnosed after rupture. Variable clinical manifestations pose the risk of misdiagnosis with a high mortality of $8.5-25 \%[6,7]$.

There is no known cause for aneurysm, but there are several risk factors that are known of that include: family history, male gender, an age $>65$, history of smoking, high blood pressure, high cholesterol, atherosclerosis, or having a connective tissue disorder like Marfans or Ehlers-Danlos syndrome. 
The diagnosis of a superior mesenteric artery aneurysm is made by computed tomographic angiography (CTA) or magnetic resonance (MR) and sometimes via an abdominal ultrasound examination. The suspicion of an aneurysm can be raised by a calcified wall on $\mathrm{X}$-rays. In addition, the changes in the surrounding tissues and organs, like the signs of chronic pancreatitis or other events, might reveal the cause of the condition. By evaluating laboratory findings, imaging, and clinical symptoms, the factors influencing condition management should be determined and additional vascular changes might be discovered.

Imaging has a very important role not only in diagnosis but also in treatment planning because on these images is possible to determine multiple parameters for surgery like aneurysmal size, neck, access vessels, etc. Image processing software and virtual planning methods are already of great importance in medical practice. A long list could be made of either free-of-charge medical imaging software platforms, e.g., [8,9], semi-commercial, e.g., [10], or commercial ones. Characteristics used for the evaluation of such platforms could be the functionalities it offers (3D rendering, segmentation, measurements, virtual simulations, etc.), the price (from free-of-charge to very expensive), the training curve, hardware requirements, etc.

In the following paper, we propose a preoperative planning protocol, which consists of four steps, namely, high-quality 3D rendering, virtual navigation (angiography) through the arteries, automatic and semi-automatic (point and click) measurements, and 3D stent simulations. These steps are exemplified and implemented via our own (in-house) software application, CardioCTNav. The main advantages of our solutions are the quality of implementation (it is a fast, stable, and bug-free application), the price (a binary package can be provided free of charge on request), ease of use (no sophisticated and difficult segmentations are required to perform the suggested planning protocol), the brief time necessary to perform the planning (it can be completed in less than $10 \mathrm{~min}$, and it has virtually no training curve), and an intuitive user interface and simple gestures that can be learned in only a few minutes.

There are other methods currently being investigated that may be useful in preoperative planning. One such method is 3D printing. In a study by [11], the authors emphasize the importance of preoperative planning as being critical for success in the surgical suite because two-dimensional (2D) presentations of anatomical structures do not accurately portray their three-dimensional (3D) complexity, often leaving physicians ill-equipped for the procedure. In the authors' opinion, medical 3D printing could provide an innovative solution to the current constraints of preoperative planning; it is important for clinicians to develop an understanding of 3D printing technologies, as well as their uses. In their paper, the many applications of 3D printing for preoperative planning are discussed.

Another potential method for preoperative planning, and from the perspective of personalized medicine, is virtual reality (VR). In a paper published by [12], the authors investigate the potential utility of a VR simulation system in both preoperative planning and intraoperative assistance, including support during thoracoscopic sublobar resection. In their paper, they described an in-house developed virtual reality (VR) surgical navigation system using head-mounted displays (HMD).

\section{Materials and Methods}

First, the radiologist must identify and characterize the SMA aneurysms, to allow the vascular interventional radiologist or vascular surgeon to choose the appropriate treatment.

A true aneurysm is formed when all three layers are weakened and dilatation of at least 1.5 times the normal diameter is observed. At the same time, a pseudoaneurysm is usually caused by vessel injury; this is the collection of blood between one or two layers and the adjacent parenchyma.

It is easy to detect large SMA aneurysms on axial section images. However, in the case of small aneurysms, MPR, maximum intensity projection, and 3D-rendered images are the best choice for visualization. In the CT angiographic report, the SMA aneurysms would be described as saccular or fusiform. In this case, the artery should be inspected carefully to 
identify a concomitant dissection. Peripheral low attenuation within the aneurysmal sac is seen with a mural thrombus. It was observed that true aneurysms have continuous wall calcifications and are often incidental. As for the pseudoaneurysms, they usually manifest symptomatically and can be caused by tumor erosion, trauma, or inflammation. Findings on CT images include extensive mural thrombus, adjacent hemorrhage, and discontinuous wall calcification. Inflammatory fat stranding can be seen near a pseudoaneurysm in patients with pancreatitis. Important information about the aneurysm's location, size, branch vessel involvement, the presence of rupture, and collateral vessel circulation is provided via multidetector CT scans.

Vascular surgeons and/or interventional radiologists and/or GI surgeons are involved in mesenteric artery aneurysm cases.

Sometimes, for patients with acute abdominal pain, a single venous phase scan is performed as SMA abnormalities may not be suspected. However, this does not mean that it is not possible to evaluate the SMA because the spatial resolution of current-generation multidetector CT scanners enables the identification of SMA abnormalities in the venous phase if the artery is inspected carefully in all imaging planes [13].

When an acute SMA abnormality is suspected, narrow detector sections $(<1 \mathrm{~mm})$, thin reconstruction sections ( $3 \mathrm{~mm}$ for axial images and $0.75 \mathrm{~mm}$ for two- or three-dimensional (2D or 3D) rendering), rapid infusion of intravenous contrast material $(5 \mathrm{~mL} / \mathrm{s})$, and imaging in the arterial and venous phases are necessary to identify lesions in the SMA and to characterize the extent of vascular, gastrointestinal, and mesenteric complications. Post-processed sagittal and coronal two-dimensional multiplanar reformations (MPRs) should be rendered routinely, and 3D rendering at an independent workstation facilitates identification and characterization of SMA abnormalities that are missed on axial images, particularly in the more distal branches. The celiac and SMA origins should be inspected on sagittal MPR images, and the distal branches of the SMA should be examined on coronal MPR images [13].

The first major branch of the SMA is the inferior pancreaticoduodenal artery. Within the mesenteric root, the SMA branches into approximately four to six jejunal and nine to 13 ileal arteries arising on the left side. The right-sided branches include the middle colic, right colic, and ileocolic arteries. The branching pattern of the colonic arteries often varies. All these blood vessels should be investigated carefully on CT angiographic images.

We used an in-house custom-made platform named CardioCTNav in order to investigate all these anatomical details and to perform surgery planning.

CardioCTNav is a cross-platform (Linux, macOS, Windows) in-house medical imaging software application based on open-source technologies- $\mathrm{C}++$ programming language, Qt [14], VTK [15], DICOM for VTK [16], CTK [17]. It is a fast and stable solution, easy to learn and to use, developed by authors and offering them complete control over the implementation of the protocol described in the following sections.

Using CardioCTNav and starting from CT data, the surgery planning procedure consists of the following steps:

- 3D reconstruction and visualization of arteries (see Figures 1 and 5)

- Virtual angiography-virtual 3D navigation through the mesenteric artery and aneurysm zone (see Figures 2 and 6-9)

- Automatic measurements of the artery diameter in "healthy" zones and aneurysm zone (see Figure 3 and Table 1)

- Stent simulation-diameter and length, 3D visualization (see Figure 4) 


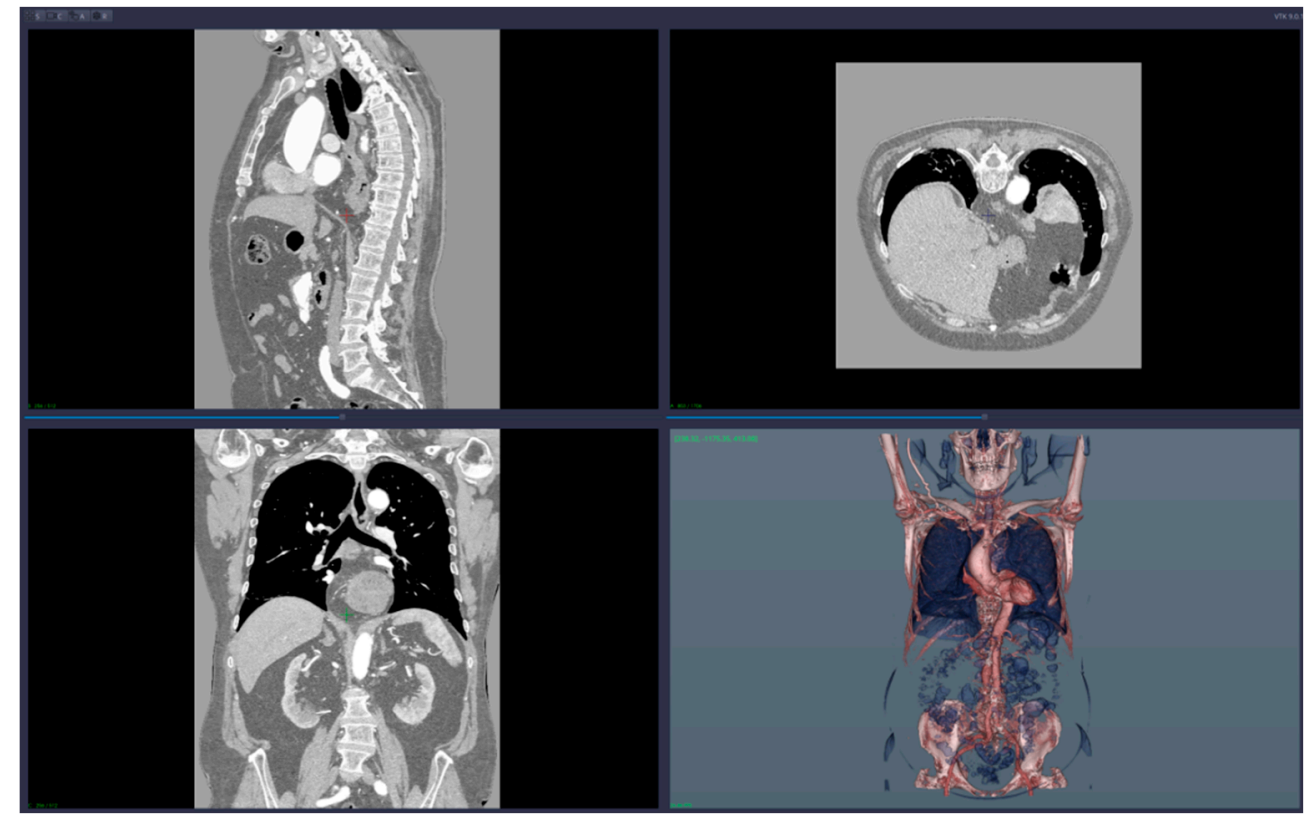

Figure 1. Images of the 3D CT reconstruction and 2D sections.

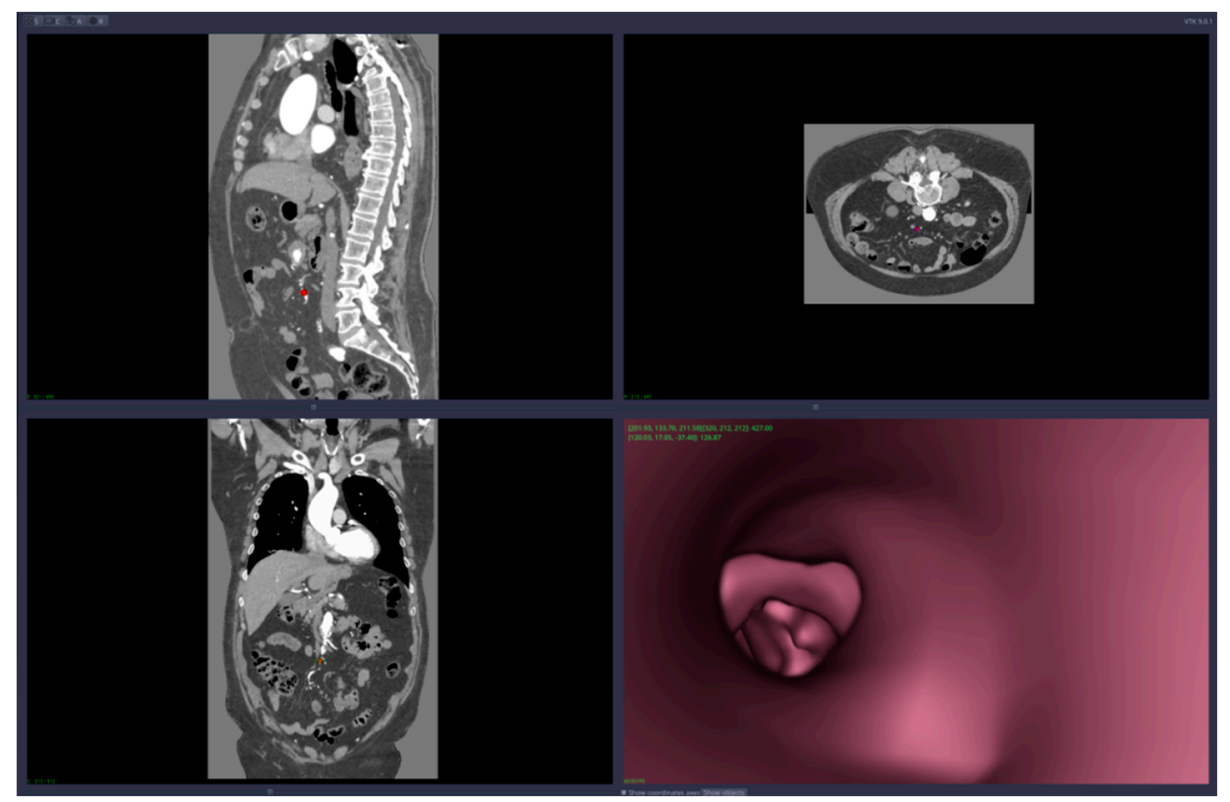

Figure 2. Virtual angiography- "normal" zone of the mesenteric artery, before the aneurysm (direction is opposite to the blood flow). 


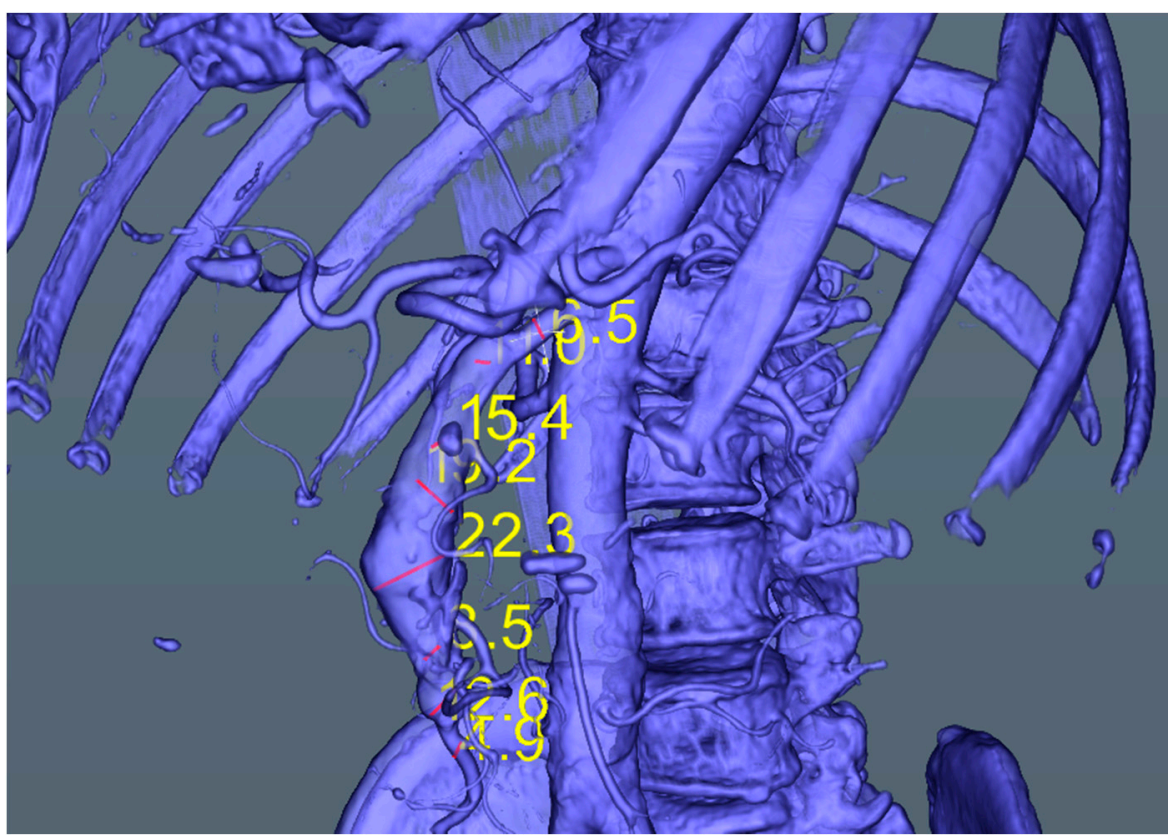

Figure 3. Measurements in the virtual 3D scene along the mesenteric artery aneurysm.

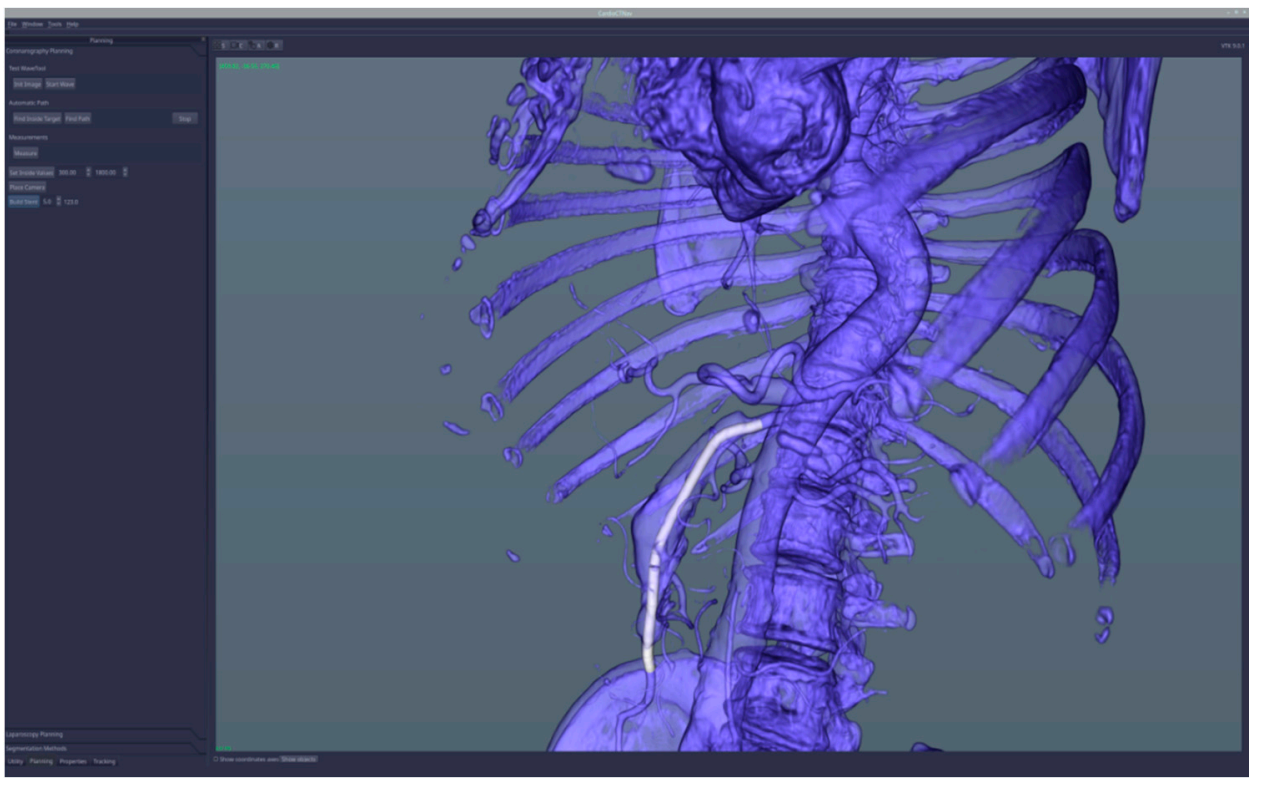

Figure 4. Stent placement-5 mm diameter, $123 \mathrm{~mm}$ length.

Table 1. Automated measurements along the aneurysm, opposite the direction of the blood flow, starting and ending with "healthy" zones.

\begin{tabular}{ccc}
\hline Entry Point-Healthy Zone & Aneurysm Zone & Exit Point-Healthy Zone \\
\hline 4.9 & 12.613 .522 .319 .215 .411 .0 & 6.5 \\
\hline
\end{tabular}




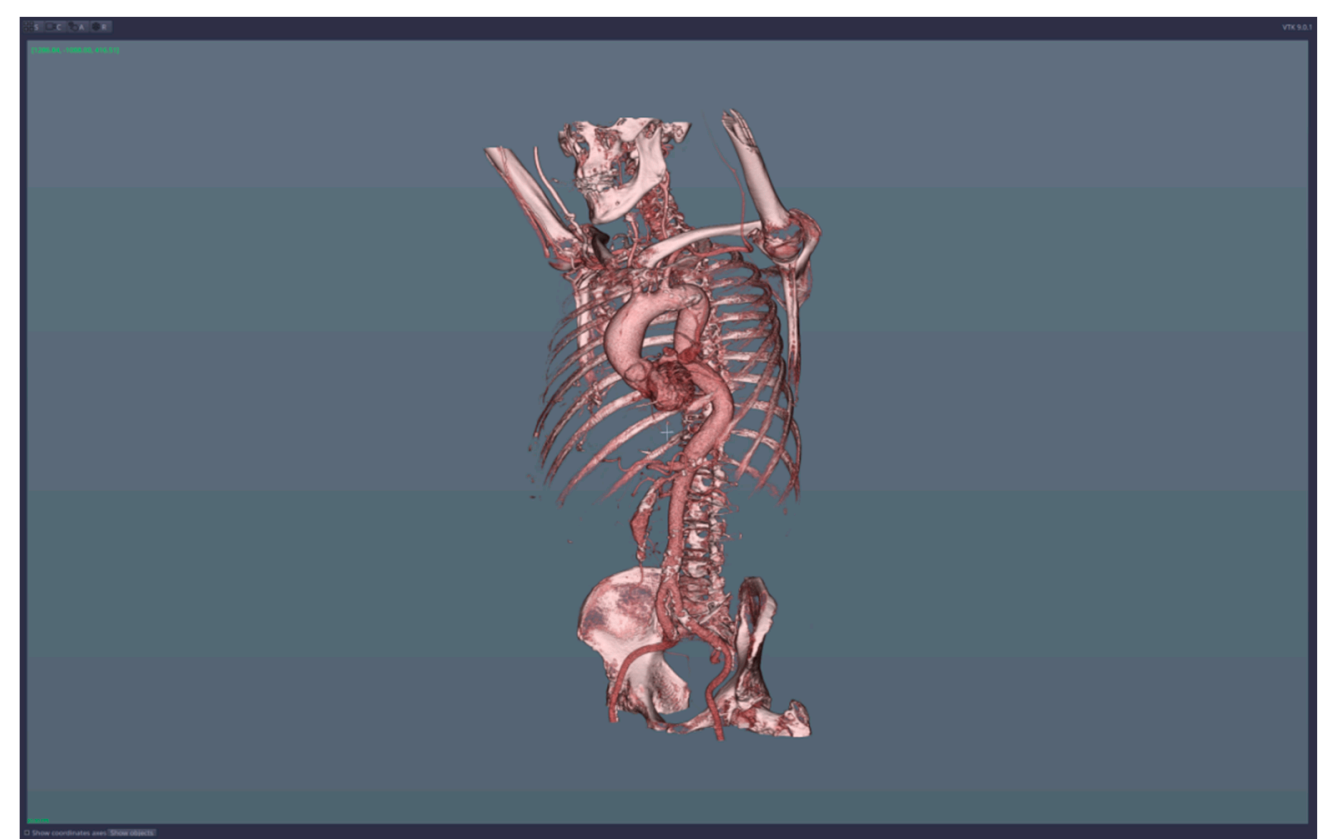

Figure 5. Images of the 3D reconstruction of main arteries and mesenteric artery aneurysm.

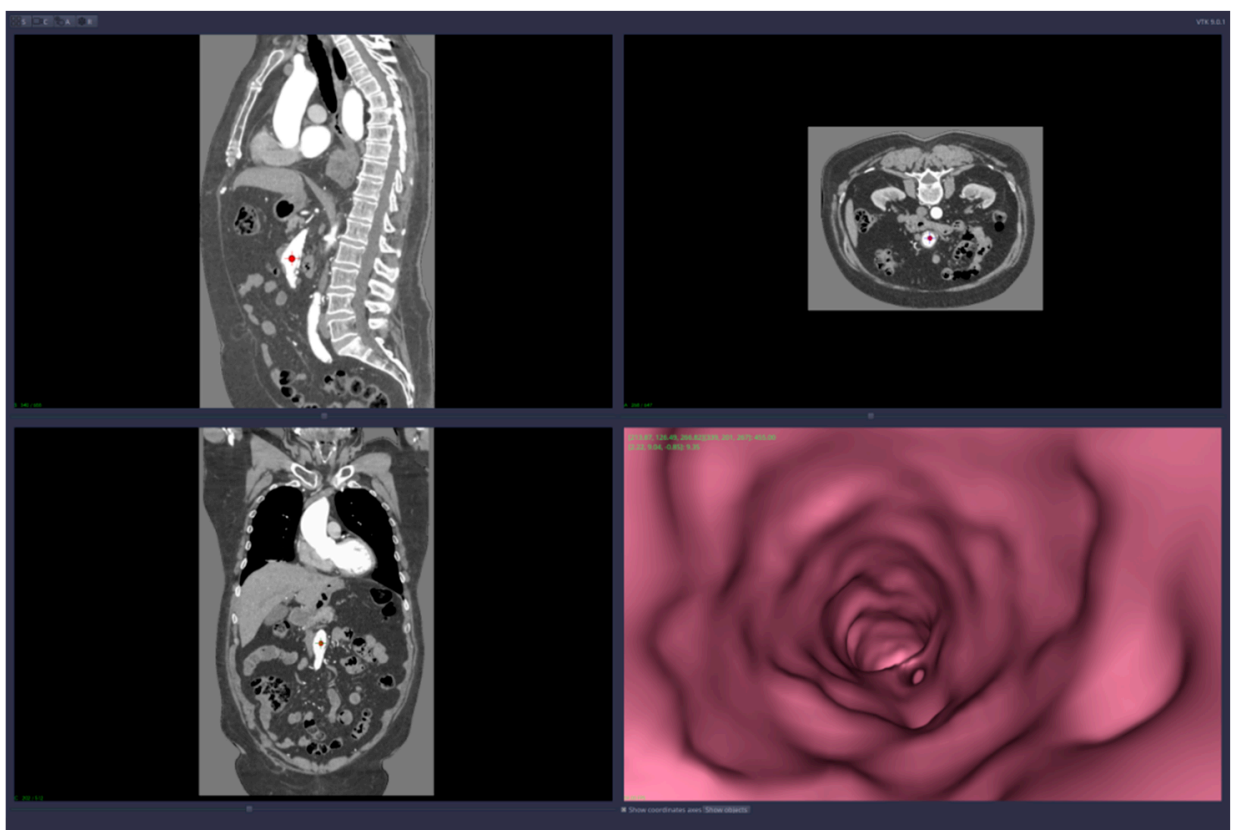

Figure 6. Virtual angiography-inside the mesenteric artery aneurysm (direction is opposite to the blood flow). 


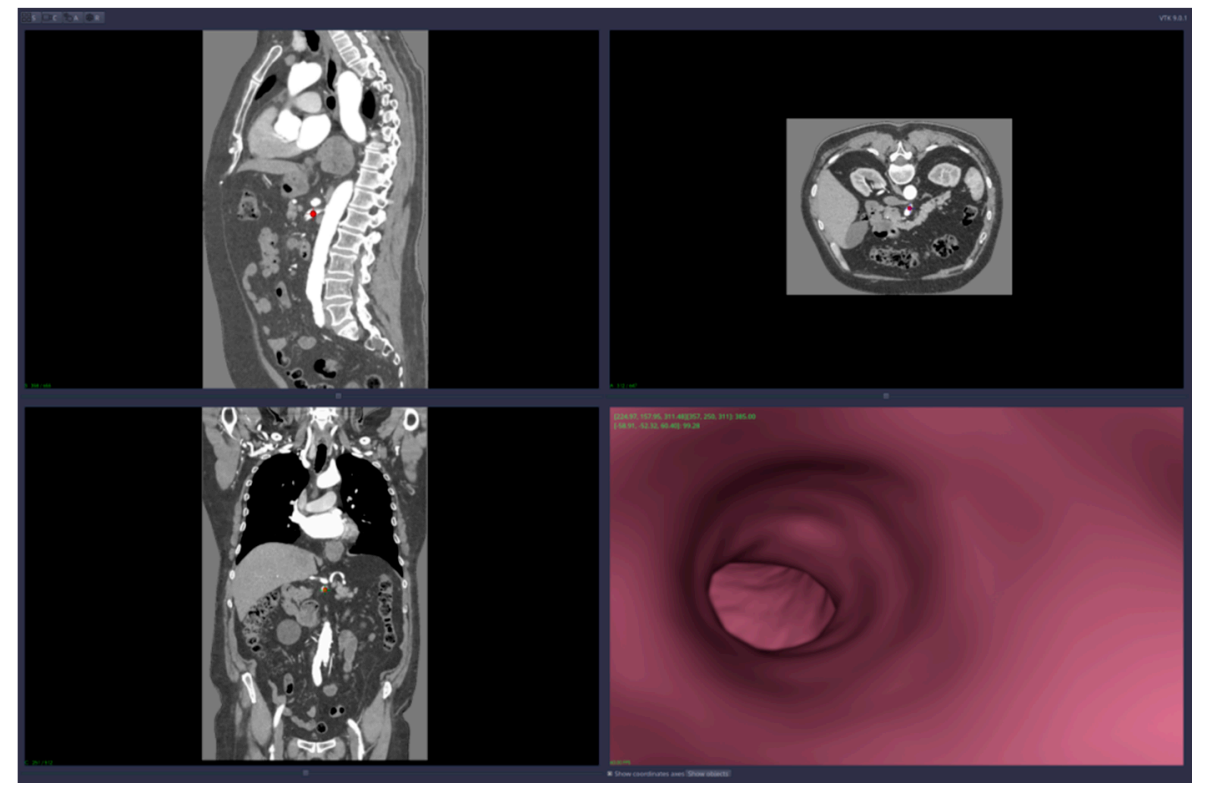

Figure 7. Virtual angiography— "normal" zone of the mesenteric artery, after the aneurysm (direction is opposite to the blood flow).

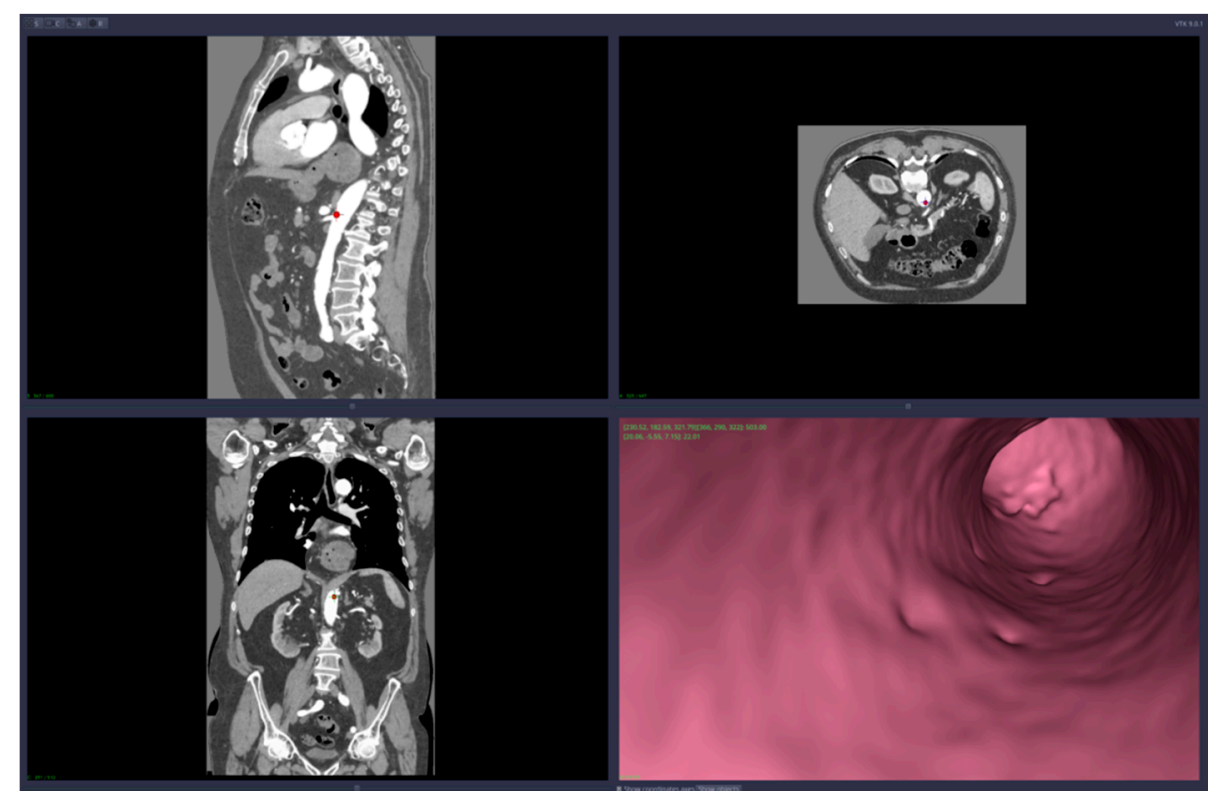

Figure 8. Virtual angiography-exit from the mesenteric artery into the aorta (direction is opposite to the blood flow). 


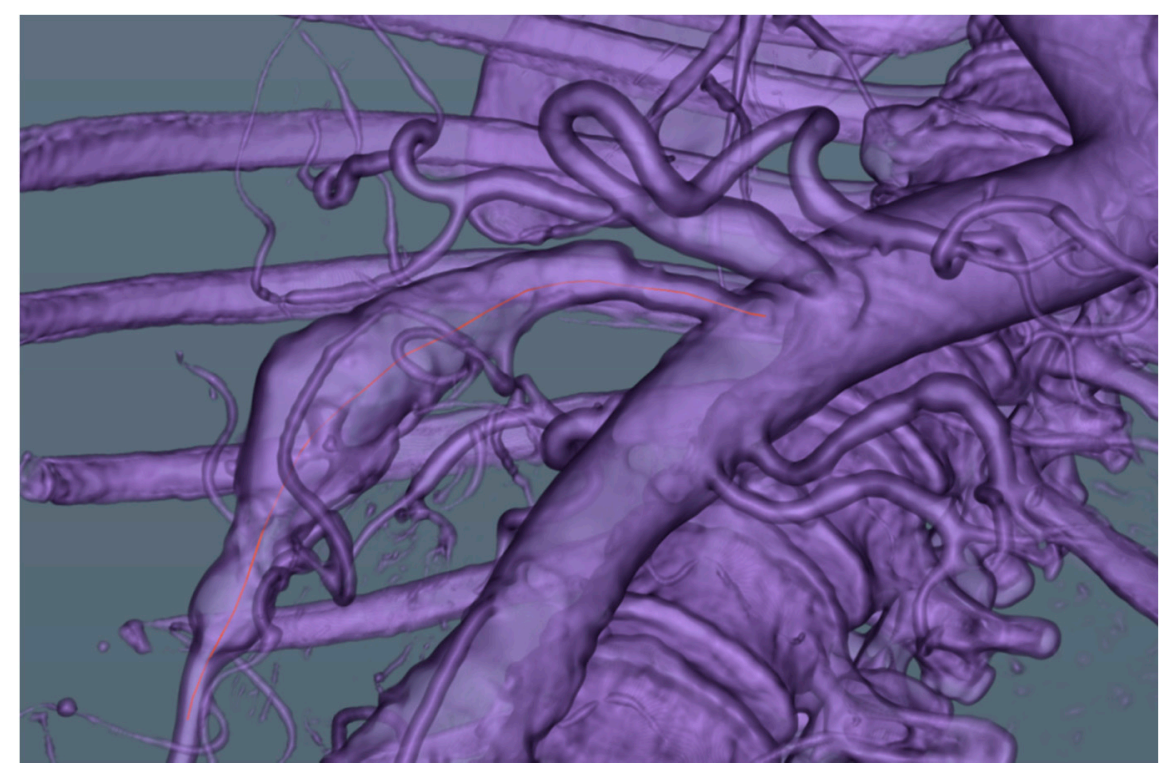

Figure 9. Virtual angiography trajectory- "procedure" started opposite to the blood flow, bottom-up, with the final point into the aorta (direction is opposite to the blood flow).

The 3D reconstruction and the visualization of arteries are based on the concept of a transfer function, which translates Hounsfield units from the CT data into colors and levels of opacity for the 3D-rendering scene. CardioCTNav implements a "freehand" drawing mode of the transfer function shapes; in this way, it allows fine-tuning in the selection of the tissues that are visualized (this fine-tuning is necessary for virtual navigation/angiography).

The CardioCTNav software platform was able to distinguish between the zones that were both inside and outside the arteries and to detect the artery walls. Target points can be chosen by the user interacting with the 2D sections; they are colored according to the zone where they are placed (outside or inside the artery). The platform is also able to detect an "inside" zone closest to a point that is initially placed in an "outside" zone.

Virtual angiography means that the user is able to navigate inside the artery using only the computer mouse device. The navigation starts from a point chosen by the user and the virtual camera is forced to remain inside the artery walls. The algorithm that implements this restriction on the virtual camera is based on collision detection and resolution directly on voxels (no segmentation is required). The path of the virtual angiography can be saved and used later, in other planning sessions.

The automatic measurements are in fact the values of the artery diameter computed automatically in points either chosen by the user or along the virtual angiography path.

The stent is generated automatically along a virtual angiography path and its diameter is decided by the user.

\section{Results}

\subsection{The Characterization of the Aneurysm and of the Anatomy on Real Patient CT Data}

We applied the suggested pre-operatory planning procedure on CT data from a male patient aged 74 . The scans were performed using a Siemens Somatom go. Top 128 slice machine [18], with contrast substance (ULTRAVIST 370 I.V.). The series used for planning took 647 slices at a distance of $0.99 \mathrm{~mm}$ each. The reconstructed 3D image had $655 \times 512 \times 647$ voxels.

The results can be seen in Figures 1-9 and Table 1:

- 3D reconstruction-various tissues, Figure 1; arteries and mesenteric artery aneurysm, Figure 5

- Virtual angiography—Figures 2 and 6-9 
- Measurements-Figure 3 and Table 1

- Stent simulation (diameter, length)-Figure 4.

As you can determine from those figures, this particular aneurysm included the whole main part of the superior mesenteric artery, was fusiform in shape and involved all small intestinal branches, which makes the cases difficult to repair.

In addition, the stent graft required to cover the whole dilated area was $5 \mathrm{~mm}$ in diameter and $123 \mathrm{~mm}$ in length. We used as an example those stents available for renal artery stenting (for example, the Herculink Elite ${ }^{\circledR}$ stent made by Abbott Vascular, Santa Clara, CA, USA, with dimensions of $7.0 \times 15 \mathrm{~mm}, 135 \mathrm{~mm}$ ).

\subsection{Planning Follow-Up for Surgery Procedures}

Our vascular surgeon considered two main methods to repair a mesenteric aneurysm: open surgery or endovascular repair [19]. In the following paragraphs, we will briefly explain both methods. When considering a course of action, the vascular surgeon will decide the best method for a particular patient based on multiple factors, like the presence of collaterals, patient frailty, etc. However, both methods require a tubular prosthetic material to be introduced inside the aneurysm that has to be specially tailored to the patient's physiology.

\subsubsection{Open Surgery with Aneurysmectomy and Bypass or Interposition Graft}

This method of revascularization is recommended as the first choice if bowel ischemia is present in patients undergoing an operation for rupture, or if preoperative symptoms or evaluation suggested mesenteric ischemia. More recent reports have shown good long-term results with an aneurysmectomy and bypass, or with an interposition graft [20,21].

The surgery consists of the interposition of two covered polyester stent grafts (two $7.0 \times 57 \mathrm{~mm}$ Jostent peripheral stent grafts made by Jomed, Helsingborg, Sweden) with reimplantation of multiple side branches into the polyester interposition graft (see Figure 10). The polyester graft must have a good size to match the dilated proximal and distal native SMA at the area of the anastomosis; this also makes reimplantation of the side branches easier than using an autologous vein graft $[22,23]$.

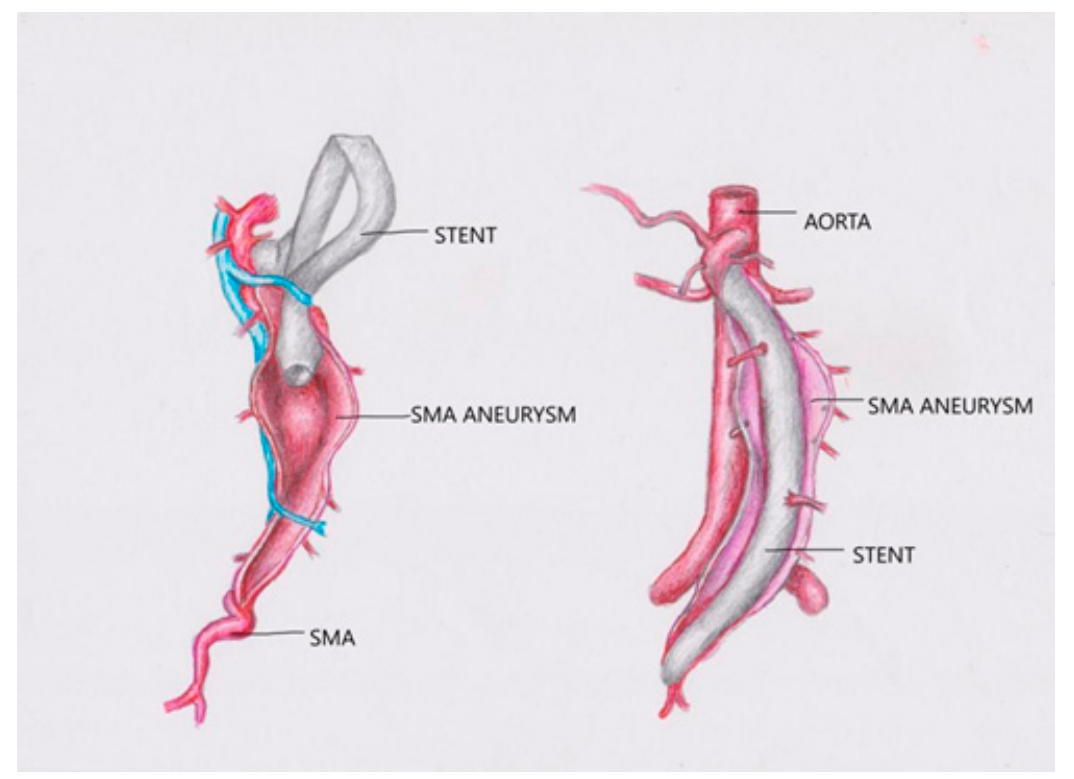

Figure 10. Endovascular repair illustration.

A clear 3D reconstruction and precise preoperative planning help the surgeon to determine exactly whether this procedure is adequate, to evaluate the type of stent technology 
needed for repair, and, most importantly, to reduce the time necessary when performing the procedure.

\subsubsection{Endovascular Aneurysm Repair (EVAR) of Superior Mesenteric Artery Aneurysm}

Endovascular treatment is safer than open surgery in patients with multiple comorbidities, such as severe cardiac or pulmonary disease. However, it should not be used when the cause of the aneurysm is suspected to be infectious. Alternative options include covered stent placement, coil embolization, liquid embolic agents, and thrombin injections [6]. Surgical options include an aneurysmectomy with grafting and/or transposition, aneurysmorrhaphy, and ligation [24].

The vascular interventional radiologist must consider several vascular factors, in addition to bowel viability, when reviewing a CT scan before the treatment of an SMA aneurysm. A femoral arterial approach is the most common, although a radial approach may be more direct when the aortic-mesenteric angle established in CT angiography is acute. Determining the number of aneurysms is important because individuals may have more than one aneurysm. The presence or absence of aneurysmal wall calcification can help to determine the patient's risk of rupture. Stone et al. [25] found that all ruptures in their study occurred in noncalcified aneurysms. An aneurysm with a small neck, distal to the SMA origin, can be treated safely with coil embolization. The aneurysm must be packed tightly with coils to promote thrombosis. With a wide-necked aneurysm, stent-assisted coil placement, in which a bare-metal stent is deployed in the parent vessel and coils are delivered into the aneurysm sac via a microcatheter passed through the interstices of the stent, is an option.

The location of the aneurysm in the SMA must be considered, because it may be difficult to place a stent in an aneurysm near the point of origin owing to the lack of an adequate landing zone [6]. The stent should extend at least $1 \mathrm{~cm}$ proximally and distally to the aneurysm [6]. Large aneurysms may not be conducive to the use of a covered stent owing to instability in a large lumen and/or inadequate landing zones. The diameter and length of the stent can usually be determined accurately from the preoperative CT angiogram and may be compared with the diagnostic angiogram obtained before endovascular intervention. Self-expanding stents are available in various sizes of $5-16 \mathrm{~mm}$ and may require different sizes of sheaths, ranging from $5 \mathrm{~F}$ to $10 \mathrm{~F}$ [6].

For vessels with a smaller diameter, lower-profile stents are available and can be placed over a 0.018-inch wire. Covered stents are preferred, to completely exclude the aneurysm and prevent an endoleak, but bare metal stents can also be used successfully [26]. The stent should extend past the aneurysm neck without covering too many jejunal or ileal branches [24]. Branches arising from the aneurysm itself also must be considered. The tortuosity of the vessel at pre-procedure CT angiography should also be noted because self-expanding covered stents are effective in tortuous vessels but balloon-expandable stents may be used in proximal straight segments [6]. CT angiography with MPR and $3 \mathrm{D}$ rendering helps to delineate the proximity of branch vessels to the aneurysm. Studies by [24] have reported covering up to three jejunoileal branches without bowel ischemia. Careful consideration of clinical presentation, gastrointestinal and/or vascular multidetector CT findings, and patient comorbidities is crucial for proper patient selection and treatment.

Another option for the side branches is to use fenestrated endovascular aortic aneurysm repair (f-EVAR), which gives more options for the endovascular treatment of pararenal aortic aneurysms. The devices are individually customized to the patient's vascular anatomy, especially to the morphology of the visceral vessels. In a typical fenestrated endovascular device design, the superior mesenteric artery (SMA) is involved, either having a large non-strut fenestration or a single wide scallop (10 $\mathrm{mm}$ in diameter).

\section{Conclusions}

By using our custom-made imaging and planning platform, CardioCTNav, we were able to provide our vascular surgeon with a clear 3D reconstruction and precise preopera- 
tive planning tool that helped him to determine exactly what procedure was most suitable, to evaluate the type of stent technology needed for repair, and, most importantly, to reduce the time necessary to perform the procedure.

Our particular case presented an SMA with an aneurysm in its entire length and multiple branches coming off of the aneurysmal segment, which made the repair more difficult. Excision and interposition grafting could have resulted in segmental bowel ischemia because of multiple patent side branches. Therefore, the vascular surgeon considered two possibilities to repair this aneurysm. The first method was similar to the reimplantation techniques of thoracoabdominal aneurysms, and involved the simulated reimplantation of multiple side branches into two polyester interposition grafts, with known good and durable results for 2 years after the operation. The polyester grafts that were selected theoretically (two $7.0 \times 58 \mathrm{~mm}$ Jostent peripheral stent grafts made by Jomed, Helsingborg, Sweden) had a 7-mm size that matched the dilated proximal and distal native SMA at the area of the anastomosis and made reimplantation of the side branches also easier than using an autologous vein graft. The second simulated option was to use baremetal renal stent grafts (for example, Herculink Elite ${ }^{\circledR}$ stents made by Abbott Vascular, Santa Clara, CA, USA, with dimensions of $7.0 \times 15 \mathrm{~mm}, 135 \mathrm{~mm}$ ).

In the future, we plan to further extend our planning module with new options like multiple stent simulations offering different sizes, blood flow calculations, etc.

Author Contributions: Conceptualization, C.I.D., C.C., R.T.P. and D.D.; methodology, D.D., R.T.P., C.I.D. and S.M.C.; software, C.C., L.D.P. and L.F.B.; validation: C.G.T. and D.M.C.; formal analysis, R.S.; investigation, M.F.T.; resources, D.D.; data curation, R.S.; writing—original draft preparation, C.C., S.M., R.T.P. and D.D.; writing, D.D. and C.G.T.; visualization: S.M.C. and M.F.T.; supervision, C.I.D. and D.D. All authors have read and agreed to the published version of the manuscript.

Funding: This research received no external funding.

Institutional Review Board Statement: Not applicable.

Informed Consent Statement: Informed consent was obtained from all subjects involved in the study.

Conflicts of Interest: The authors declare no conflict of interest.

$\begin{array}{ll}\text { Abbreviations } \\ \text { SMA } & \text { superior mesenteric artery } \\ \text { MPR } & \text { multiplanar reconstruction } \\ \text { CT } & \text { computer tomography } \\ \text { GI } & \text { gastrointestinal } \\ \text { CTA } & \text { computed tomographic angiography } \\ \text { EVAR } & \text { endovascular aneurysm repair } \\ \text { f-EVAR } & \text { fenestrated endovascular aortic aneurysm repair }\end{array}$

\section{References}

1. Ullery, B.W.; Lee, G.K.; Lee, J.T. Shuttering of the superior mesenteric artery during fenestrated endovascular aneurysm repair. J. Vasc. Surg. 2014, 60, 900-907. [CrossRef] [PubMed]

2. Yongcheng, X.; Yukun, L. The Fate of the Superior Mesenteric Artery in Fenestrated Endovascular Repair of Complex Abdominal Aortic Aneurysms. Vasc. Endovasc. Rev. 2019, 2, 38-39. [CrossRef]

3. Greenberg, R.K.; Haulon, S.; Lyden, S.P.; Srivastava, S.D.; Turc, A.; Eagleton, M.J.; Sarac, T.P.; Ouriel, K. Endovascular management of juxtarenal aneurysms with fenestrated endovascular grafting. J. Vasc. Surg. 2004, 39, 279-287. [CrossRef] [PubMed]

4. Tkalčić, L.; Budiselić, B.; Kovačević, M.; Knežević, S.; Kovačić, S.; Miletić, D.; Tomulić, V.; Kuhelj, D. Endovascular Management of Superior Mesenteric Artery (SMA) Aneurysm-Adequate Access is Essential for Success—Case Report. Pol. J. Radiol. 2017, 82, 379-383. [CrossRef] [PubMed]

5. Balderi, A.; Antonietti, A.; Ferro, L.; Peano, E.; Pedrazzini, F.; Fonio, P.; Grosso, M. Endovascular treatment of visceral artery aneurysms and pseudoaneurysms: Our experience. La Radiol. Med. 2012, 117, 815-830. [CrossRef] [PubMed]

6. Hemp, J.H.; Sabri, S.S. Endovascular Management of Visceral Arterial Aneurysms. Tech. Vasc. Interv. Radiol. 2015, 18, 14-23. [CrossRef] [PubMed] 
7. Cochennec, F.; Riga, C.; Allaire, E.; Cheshire, N.; Hamady, M.; Jenkins, M.; Kobeiter, H.; Wolfe, J.; Becquemin, J.; Gibbs, R. Contemporary Management of Splanchnic and Renal Artery Aneurysms: Results of Endovascular Compared with Open Surgery from Two European Vascular Centers. Eur. J. Vasc. Endovasc. Surg. 2011, 42, 340-346. [CrossRef] [PubMed]

8. 3D Slicer Image Computing Platform. Available online: https://www.slicer.org/ (accessed on 10 October 2021).

9. The Medical Imaging Interaction Toolkit (MITK). Available online: https://www.mitk.org (accessed on 10 October 2021).

10. OsiriX Viewer. Available online: http:/ / www.osirix-viewer.com (accessed on 10 October 2021).

11. Alemayehu, D.G.; Zhang, Z.; Tahir, E.; Gateau, D.; Zhang, D.F.; Ma, X. Preoperative Planning Using 3D Printing Technology in Orthopedic Surgery. BioMed Res. Int. 2021, 2021, 7940242.

12. Ujiie, H.; Yamaguchi, A.; Gregor, A.; Chan, H.; Kato, T.; Hida, Y.; Kaga, K.; Wakasa, S.; Eitel, C.; Clapp, T.R.; et al. Developing a virtual reality simulation system for preoperative planning of thoracoscopic thoracic surgery. J. Thorac. Dis. 2021, 13, 778. [CrossRef] [PubMed]

13. Ghodasara, N.; Liddell, R.; Fishman, E.K.; Johnson, P.T. High-Value Multidetector CT Angiography of the Superior Mesenteric Artery: What Emergency Medicine Physicians and Interventional Radiologists Need to Know. Radiographics 2019, 39, 559-577. [CrossRef] [PubMed]

14. Qt Framework. Available online: https://www.qt.io/ (accessed on 10 October 2021).

15. Visualization Toolkit(VTK). Available online: https://vtk.org/ (accessed on 10 October 2021).

16. DICOM for VTK. Available online: https://dgobbi.github.io/vtk-dicom/ (accessed on 10 October 2021).

17. The Common Toolkit(CTK). Available online: https:// commontk.org (accessed on 10 October 2021).

18. Siemens Somatom Go Top. Available online: https://www.siemens-healthineers.com/computed-tomography/single-source-ctscanner/somatom-go-top (accessed on 1 November 2021).

19. Carr, S.C.; Mahvi, D.M.; Hoch, J.R.; Archer, C.W.; Turnipseed, W.D. Visceral artery aneurysm rupture. J. Vasc. Surg. 2001, 33, 806-811. [CrossRef] [PubMed]

20. Ghariani, M.Z.; Georg, Y.; Ramirez, C.; Lebied, E.; Gaudric, J.; Chiche, L.; Kieffer, E.; Koskas, F. Long-Term Results of Surgical Treatment of Aneurysms of Digestive Arteries. Ann. Vasc. Surg. 2013, 27, 954-958. [CrossRef] [PubMed]

21. Sachdev, U.; Baril, D.T.; Ellozy, S.H.; Lookstein, R.A.; Silverberg, D.; Jacobs, T.S.; Carroccio, A.; Teodorescu, V.J.; Marin, M.L. Management of aneurysms involving branches of the celiac and superior mesenteric arteries: A comparison of surgical and endovascular therapy. J. Vasc. Surg. 2006, 44, 718-724. [CrossRef] [PubMed]

22. Arghami, A.; Krajewski, A.C.; Gloviczki, P.; Leduc, C.; Lin, P.T. Open staged repair of splenic and extensive superior mesenteric artery aneurysms. J. Vasc. Surg. 2019, 69, 1252-1256. [CrossRef] [PubMed]

23. Carr, S.C.; Pearce, W.H.; Vogelzang, R.L.; McCarthy, W.J.; Nemcek, A.A.; Yao, J.S. Current management of visceral artery aneurysms. Surgery 1996, 120, 627-634. [CrossRef]

24. Jiang, J.; Ding, X.; Su, Q.; Zhang, G.; Wang, Q.; Jian, W.; Wang, Z.; Hu, S. Therapeutic management of superior mesenteric artery aneurysms. J. Vasc. Surg. 2011, 53, 1619-1624. [CrossRef]

25. Stone, W.M.; Abbas, M.; Cherry, K.J.; Fowl, R.J.; Gloviczki, P. Superior mesenteric artery aneurysms: Is presence an indication for intervention? J. Vasc. Surg. 2002, 36, 234-237. [CrossRef]

26. Geana, R.C.; Pavel, P.; Nayyerani, R.; Kulcsar, I.; Tulin, A.; Honciuc, O.; Balescu, I.; Bacalbasa, N.; Stiru, O.; Iliescu, V.A.; et al. Successfully superior mesenteric artery stenting in operated type A aortic dissection complicated with delayed mesenteric malperfusion. SAGE Open Med. Case Rep. 2021, 9, 2050313X211021184. [CrossRef] [PubMed] 